\title{
Long-term studies of the optical components in the ZEUS calorimeter using a moving ${ }^{60}$ Co source ${ }^{\text {t }}$
}

\author{
I. Bohnet *, R.-P. Feller, N. Krumnack ${ }^{1}$, E. Möller, H. Prause, H. Salehi, K. Wick \\ Institut für Experimentalphysik, University of Hamburg, Germany
}

\section{A R T I C L E I N F O}

\section{Article history:}

Received 21 January 2008

Received in revised form

22 October 2008

Accepted 27 October 2008

Available online 12 November 2008

\section{Keywords:}

Electromagnetic and hadronic calorimeters

Scintillator

Wavelength shifter

Ageing

Radiation damage

\begin{abstract}
A B S T R A C T
The response of the ZEUS sampling calorimeter has been studied over a period of 17 years using a moving ${ }^{60} \mathrm{Co}$ source. The aim was to monitor the stability of the calorimeter with respect to radiation damage, ageing and mechanical damage. The measurements started in 1990 during the installation of the first calorimeter modules and ended in 2007 after the last HERA run. Within the experimental uncertainties, the longitudinal uniformity of the polymethylmethacrylate (PMMA) based wavelength shifters (Y7) did not change. There is also no evidence for radiation damage in the polystyrene (PS) based scintillators (SCSN-38) due to particle losses of the electron and proton beams. On the other hand, the investigations clearly indicate that the attenuation length of the SCSN-38 scintillators decreased continuously over the years. A comparison with data obtained from a test calorimeter showed that the same effect observed in the ZEUS calorimeter after 17 years would require a uniform exposure of all scintillators by a dose of $(2.8 \pm 1) \mathrm{kGy}$ of $\gamma$ rays. However, it can be excluded that the degradation observed in the ZEUS calorimeter can be attributed to the radioactivity of the uranium plates of the calorimeter. Thus we expect that the decrease of the attenuation length is due to ageing of the scintillators. The ageing effects observed in SCSN-38 are compared to data taken from the literature.
\end{abstract}

(c) 2008 Elsevier B.V. All rights reserved.

\section{Introduction}

The task of the ZEUS detector at the lepton-proton collider HERA at DESY was to provide highly reliable and precise data of ep final states for a period of more than 15 years. A main component of the ZEUS detector was the high resolution sampling calorimeter consisting of alternating layers of depleted uranium (DU) and plastic scintillator tiles, which were read out by wavelength shifting light guides and photomultipliers. During the running period of HERA, the ZEUS experiment required a precise measurement of particle energies from ep scattering with an calibration accuracy of $\pm 1 \%$. In order to guarantee a stable detector performance over the running period of more than 15 years questions concerning ageing and radiation resistance of the scintillators and wavelength shifters (WLSs) but also concerning the durability of electronic components had to be addressed. Besides radiation from the DU plates radiation due to particle losses was of concern especially for components close to the circulating beams. Several active and passive monitoring systems were therefore installed, for example an automatic beam dump

\footnotetext{
Supported by the German Ministry for Education, Science, Research and Technology (BMBF) under the Contract 05 7HH 19P.

*Corresponding author. Now at DESY, Notkestr. 85, D-22607 Hamburg, Germany. Tel.: +49408998 4888; fax: +494089984304.

E-mail address: ilja.bohnet@desy.de (I. Bohnet).

${ }^{1}$ Now at FERMILAB, Batavia, IL, USA.
}

system, in operation since the luminosity upgrade of HERA in 2000 , to prevent high radiation doses from beam background [1]. Since the start-up of ZEUS a movable point-like $\gamma$ source $\left({ }^{60} \mathrm{Co}\right)$ was employed to investigate the uniformity of the calorimeter [2]. This monitoring system was used during the installation phase for investigations of the complete ZEUS forward and rear calorimeter in the years 1990/91, and furthermore during most of the maintenance periods on selected calorimeter components especially those close to the HERA beam pipe. No variation in the uniformity of the ZEUS calorimeter was observed, thus no irradiation damage induced by the beams could be detected. ${ }^{2}$ But indications for a slight degradation of an optical parameter of the scintillators led to special investigations in the maintenance periods 1999, 2001, 2003 and after the end of operation of HERA, in 2007, which show that the attenuation length of the SCSN-38 scintillators of the ZEUS calorimeter decreased continuously within these 17 years of observation.

\section{Description of the ZEUS calorimeter}

The ZEUS sampling calorimeter was sectioned into the forward calorimeter (FCAL), which was installed in the proton direction,

\footnotetext{
${ }^{2}$ However, severe radiation damages were detected by measurements with the ${ }^{60} \mathrm{Co}$ monitoring system in several additional calorimeter components of the ZEUS experiment [3].
} 
the barrel calorimeter (BCAL), which cylindrically surrounded the tracking devices and the rear calorimeter (RCAL) which was installed in the electron direction. The calorimeter had a modular structure consisting of 80 modules in total. F/RCAL were separated into left and right halves and could be retracted sideways. The F/RCAL modules were transversally subdivided into towers with a cross-sectional area of $20 \times 20 \mathrm{~cm}^{2}$. Thirty-two identical modules surrounded as so-called BCAL the tracking devices which varied in width from $24 \mathrm{~cm}$ at the front to $43 \mathrm{~cm}$ at the outer radius. The transverse subdivision of the BCAL into towers was similar to F/RCAL. The calorimeter modules were subdivided longitudinally into electromagnetic sections (EMC) and hadronic sections (HAC). The electromagnetic sections are vertically subdivided in two and four sections for RCAL and FCAL, respectively. The sections at the top and bottom of the calorimeter had no vertical subdivision of the electromagnetic sections (HACO).

The 5917 sections of the calorimeter consisted of alternating layers of absorber and scintillator material configured in a sandwich construction. The absorber material was DU, which has a density of $\approx 19 \mathrm{~g} / \mathrm{cm}^{3}$. Each uranium layer with a thickness of $3.3 \mathrm{~mm}$ was embedded in a thin steel box $(0.2 \mathrm{~mm}$ for electromagnetic and $0.4 \mathrm{~mm}$ for hadronic sections). Polystyrene based plastic scintillators (SCSN-38, see Ref. [4]) with a thickness of $2.6 \mathrm{~mm}$ were used as the active material. The size of the scintillators was $49.4 \times 191 \mathrm{~mm}^{2}$ for the electromagnetic section (EMC), and $199.4 \times 186 \mathrm{~mm}^{2}$ for the first hadronic section (HAC1), and $199.4 \times 181 \mathrm{~mm}^{2}$ for the second hadronic section (HAC2). The scintillators were wrapped in Tyvek paper with a reflection pattern to compensate the non-uniform response at the edge of the scintillator. Each calorimeter section was connected with the right and left WLS based on polymethylmethacrylate (Y7, see Ref. [4]). The WLS bars have a thickness of $2.0 \mathrm{~mm}$ and lengths up to $2 \mathrm{~m}$, the active areas are $24 \mathrm{~cm}$ for the electromagnetic and about $64 \mathrm{~cm}$ for the hadronic sections. The WLSs were read out by photomultipliers (PMT) of type Philips XP 1911 for the electromagnetic and Hamamatsu R-580 for the hadronic sections. The segmentation of the ZEUS calorimeter resulted in a total of 6526 photomultipliers for the electromagnetic sections and 5308 for the hadronic sections for all calorimeter sections. A detailed description of the ZEUS sampling calorimeter can be found in Ref. [5].

\section{The ${ }^{60}$ Co monitoring system and data analysis}

The principle of the ${ }^{60} \mathrm{Co}$ monitoring system is based on a point-like ${ }^{60} \mathrm{Co}$ source (emitting $\gamma$ rays of 1.17 and $1.33 \mathrm{MeV}$ ) which can be moved parallel to the WLS bars of the investigated calorimeter section. ${ }^{3}$ The source is encapsulated in a steel tube at the tip of a special wire. Brass tubes, which are incorporated into the WLS light guide assemblies on each tower side of the calorimeter, allow to guide the source forwards and backwards. The permanently installed source wire guiding system allows to repeat ${ }^{60} \mathrm{Co}$ scans with a reproducibility of $1 \%$. The $\gamma$ rays emitted by the ${ }^{60} \mathrm{Co}$ source cause a local energy deposition at the edges of the scintillators. The light emitted at both edges of the scintillators was detected in both photomultiplier tubes as can be seen in Fig. 1.

A total of four response functions $I_{k j}(z)$ was obtained by moving the source along both sides of each calorimeter section. The first index $k$ refers to the $\operatorname{side}^{4}$ of the calorimeter (right $R$ or left $L$ ) where the source is located, while the second index $j$ indicates the

\footnotetext{
${ }^{3}$ In the following we will report only about studies on FCAL and RCAL sections.

${ }^{4}$ In the following text right and left sides are defined for a person looking towards the interaction point.
}

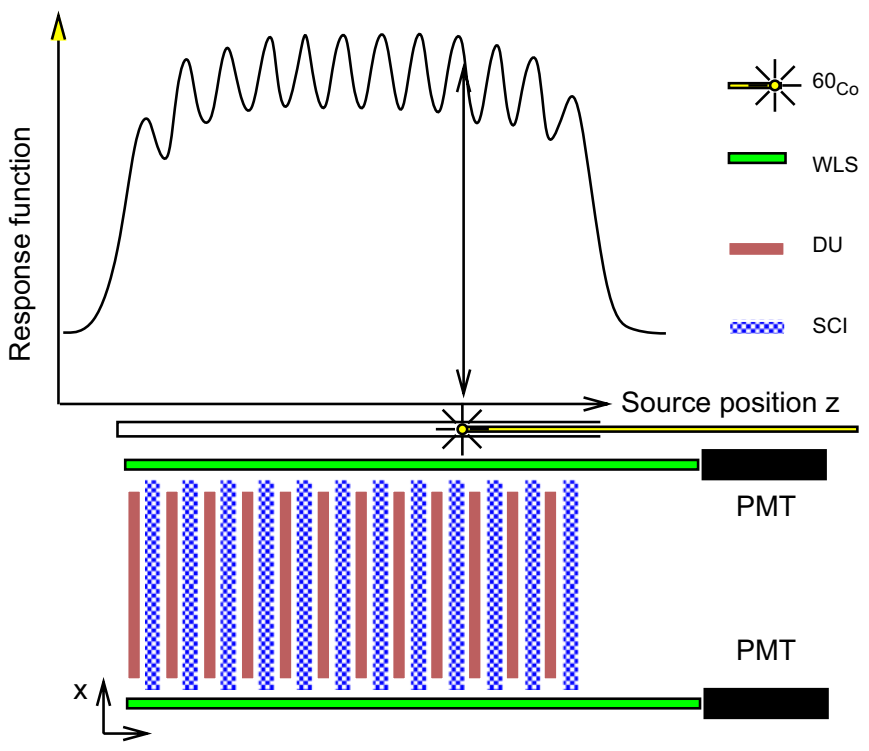

Fig. 1. Top view on a calorimeter section scanned by a ${ }^{60} \mathrm{Co}$ source (bottom) and the response function as a function of the source position (top).

position of the photomultiplier tube, and $z$ denotes the position of the source. The response functions were normalized as

$\hat{I}_{k j}(z)=\frac{I_{k j}(z)-U_{j}}{U_{j}}$

where the reference signal $U_{j}$ is derived from the radioactivity of the uranium plates measured by photomultiplier $j$ of the calorimeter. The four response functions of two ${ }^{60} \mathrm{Co}$ measurements $\hat{I}_{R R}(z), \hat{I}_{R L}(z), \hat{I}_{L R}(z), \hat{I}_{L L}(z)$ can be described by a model: when we assume that the ${ }^{60} \mathrm{Co}$ source is located on the right side of the calorimeter, the light yields registered by the right $\left(\hat{I}_{R R}\right)$ and left photomultiplier $\left(\hat{I}_{R L}\right)$ can be written as

$\hat{I}_{R R}(z)=S_{R}(z) \cdot W_{R}(z)$

$\hat{I}_{R L}(z)=S_{R}(z) \cdot T(z) \cdot W_{L}(z)$.

$S_{R}(z)$ denotes the light yield of the scintillator emitted on the side where the source is positioned and $S_{R}(z) \cdot T(z)$ is the light yield emitted on the opposite side of the scintillators. $T(z)$ represents the transmission of fluorescent light through the scintillator tile which is correlated with the attenuation length $\lambda$ of the latter. ${ }^{5}$ The transmission parameter $T(z)$ is defined as the ratio of light yields emitted at both edges of the scintillator when it is exposed to $\gamma$ rays at one end. $W_{R}(z)$ and $W_{L}(z)$ are the response functions of the right and left WLS bars. With two ${ }^{60}$ Co scans performed on both sides of the calorimeter section the transmission parameter $T$ and the ratios $S_{L} / S_{R}$ and $W_{L} / W_{R}$ for each scintillator can be determined. These parameters are from now on referred to as optical parameters.

The experimental set-up of the ${ }^{60} \mathrm{Co}$ monitoring system has been described in detail in Ref. [6]. Although the principles remained unchanged, several new components have been developed between 1990 and 2007. In the beginning, an OS9/VME based measurement system was used which was replaced in 1995 by a PC based one. However, the reproducibility of the ${ }^{60} \mathrm{Co}$ measurements was well established and checked again with each technical modification.

\footnotetext{
${ }^{5}$ An idea of the relation between the transmission parameter and the attenuation length is given in Section 5.
} 


\section{Investigations of the optical components}

Investigations with the ${ }^{60} \mathrm{Co}$ monitoring system have been performed in 1990/91 on all modules of F/RCAL before the ZEUS calorimeter was assembled. For selected sections especially those close to the HERA beam pipe these investigations have been repeated almost during every maintenance period to search for changes of the optical parameters in the ZEUS calorimeter. No effect which could be attributed to beam induced radiation damage has been observed. Within the experimental errors no variation of the attenuation length of the WLSs was detected. However, a small systematic reduction of the transmission $T$ of the scintillators became apparent.

To investigate the degradation of the transmission parameter of the scintillators in detail, ${ }^{60} \mathrm{Co}$ measurements were performed in the maintenance periods 1999, 2001, 2003 and 2007 on FCAL module 17. FCAL module 17 was shielded by other calorimeter modules from the HERA beams so that a damage due to the beams can be excluded. For the years 1990, 1999, 2001, 2003 and 2007 a common data set of four towers with 4 HAC1, 3 HAC2 and 2 EMC sections exists. Only the results concerning the hadronic sections will be discussed in the following since the geometry of the

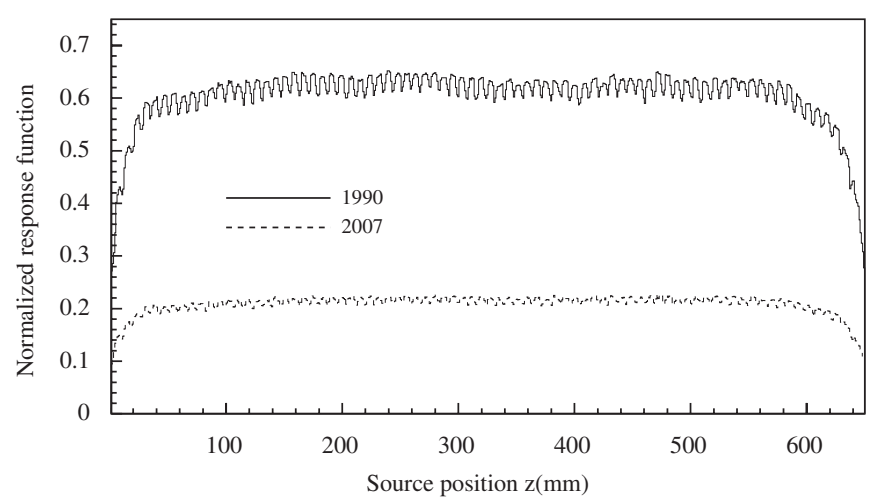

Fig. 2. Response functions of the left photomultiplier of the second hadronic section of FCAL module 17, tower 8 measured in 1990 (solid line) and in 2007 (dashed line) as a function of the source position. The ${ }^{60} \mathrm{Co}$ source was guided on the right side of the tower. Small peaks indicate the positions of the scintillators. electromagnetic sections was modified considerably during the running period of the ZEUS detector by the addition of silicon detectors in the so-called silicon gaps of the EMC sections.

Fig. 2 shows two typical response functions of a hadronic section, one from the first measurements in 1990, the second one from measurements after the HERA shut down 17 years later. The absolute size of the signals differs considerably because different ${ }^{60}$ Co sources were used in 1990 and 2007. However, the ratio of the response functions is to good approximation constant as can be seen from Fig. 3a. This indicates that the longitudinal structure of the section and the attenuation length of the WLS has not changed. The gross structure of both curves is the same as was verified by a point to point comparison. The ratios of the response functions were found to be constant at the $1-2 \%$ level, as it is demonstrated in Fig. 3b. The purely statistical uncertainty, as it was determined by repeated scans, is of the order of $1 \%$. Fig. 4 presents the optical parameters $T(z), S_{L}(z) / S_{R}(z)$ and $W_{L}(z) / W_{R}(z)$ of the hadronic sections HAC1/2 of tower 8 which were determined from ${ }^{60} \mathrm{Co}$ measurements in 1990 and 2007. The transmission $T(z)$ decreased significantly within this time, while the ratios of the WLS and scintillator response functions $W_{L}(z) / W_{R}(z)$ and $S_{L}(z) / S_{R}(z)$ show no variation with time. In order to determine the optical parameters for each scintillator an average over the mean thickness of the uranium/scintillator layers of $8 \mathrm{~mm}$ has to be performed. Fig. 5 illustrates the distribution of the transmissions for all scintillators of the sections HAC1/2, which were scanned in 1990, 1999, 2001, 2003 and 2007. The mean transmission as determined from the distributions presented in Fig. 5 is presented in Table 1. It shows a continuous decrease between 1990 and 2007 as illustrated in Fig. 6 .

\section{Damage scenarios}

The slight degradation of the transmission parameters has been observed in all sections of FCAL module 17 which were measured with the ${ }^{60} \mathrm{Co}$ source. A local mechanical damage or other accidents can be excluded as well as a possible damage induced by the electron and proton beams of HERA. Hence we assume that the same effects occurred in all scintillators of the ZEUS forward and rear calorimeter. Possible sources for the a

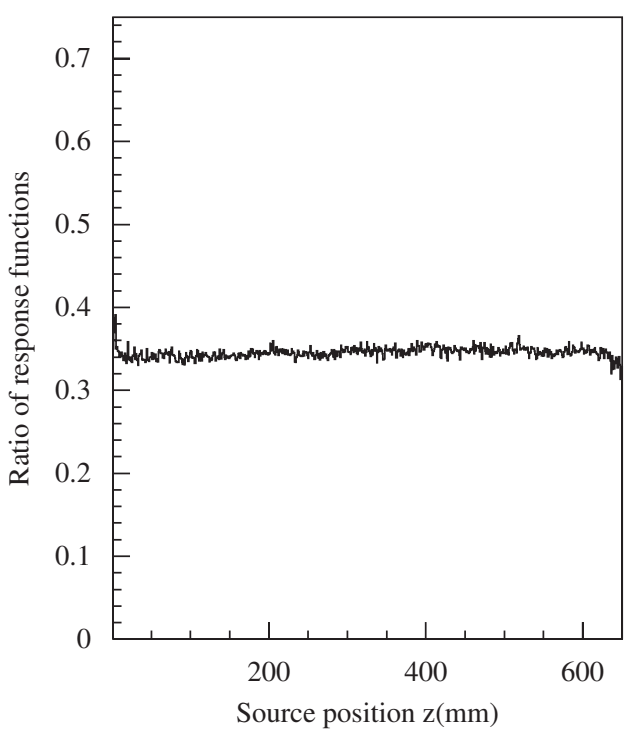

b

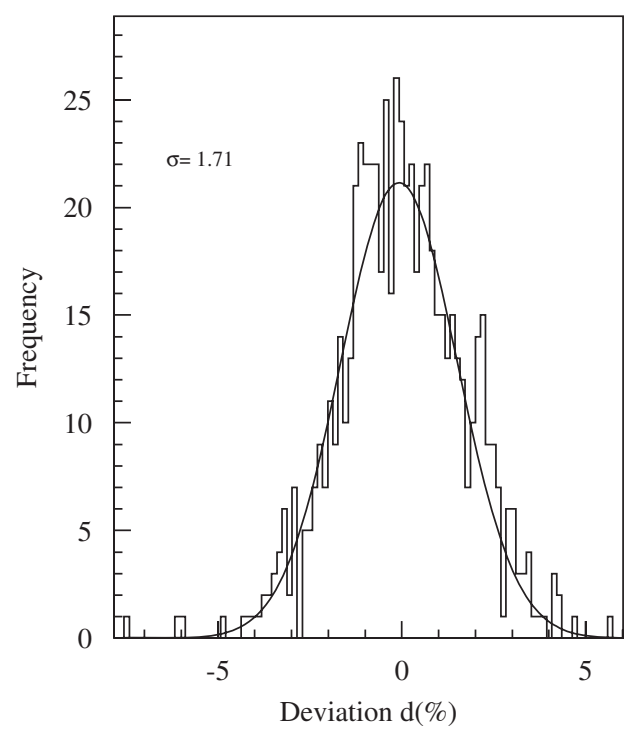

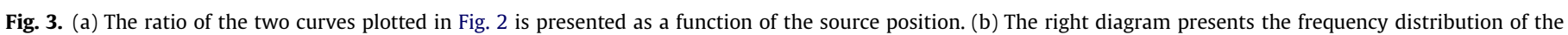
difference of both response functions divided by the corresponding mean values. 

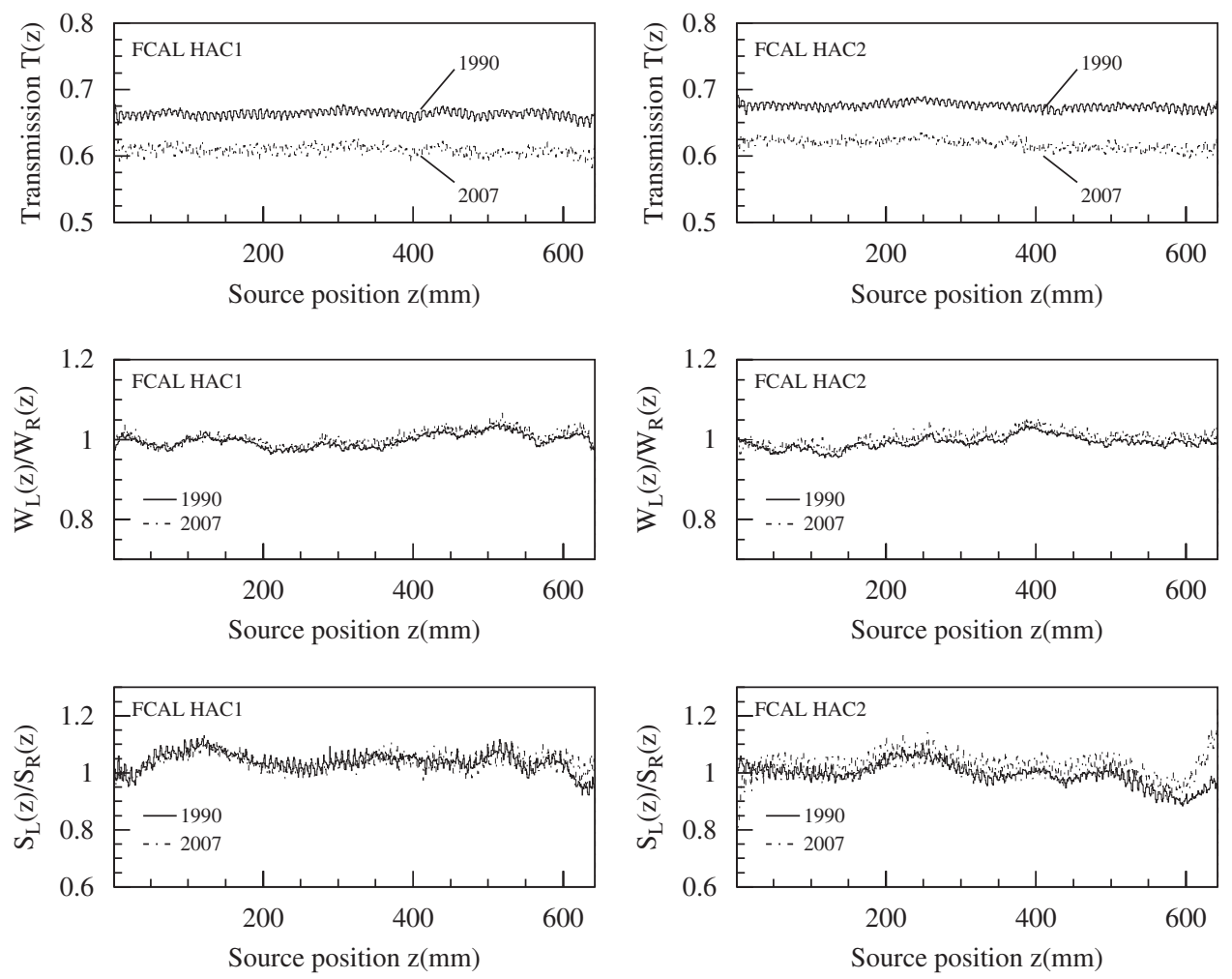

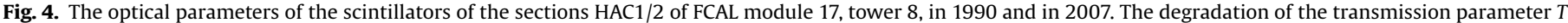
(top diagrams) within this time is clearly visible.

observed effects may be:

- ageing of the plastic scintillators,

- optical contact of the Tyvek paper on the scintillators due to humidity,

- radiation damage caused by the natural radioactivity of the DU.

Optical contact of the Tyvek paper on the scintillators cannot be completely excluded, but it seems to be very unlikely. If local humidity would be the reason for an optical contact one would not expect that the same damage effect occurs in all investigated sections. We have checked the surface of several spare scintillators which were stored in a box since 1990 . The scintillators were wrapped in the same Tyvek paper as the ones in the calorimeter. No visible change of the surface quality was observed. ${ }^{6}$

In order to discuss a possible radiation damage caused by the background radiation we first estimate the dose absorbed by the scintillators: each scintillator tile of the calorimeter is located between two DU plates which are embedded in steel boxes. ${ }^{238} \mathrm{U}$ decays via 14 steps into the stable nucleus ${ }^{206} \mathrm{~Pb}$. Outside of the uranium plates, one can detect $\gamma$ rays with energies up to $1.8 \mathrm{MeV}$, electrons with a maximum energy of $2.29 \mathrm{MeV}$ and bremsstrahlung. The energy deposition caused by the electrons in the scintillators is greatly reduced by the steel box, from the data of Ref. [7] one can estimate that the dose rate induced by the electrons in the scintillators is $2 \times 270=540 \mu \mathrm{Gy} / \mathrm{h}$. The photon background is only weakly influenced by the thin steel plates, it is about $2 \times 70=140 \mu \mathrm{Gy} / \mathrm{h}$. The total dose rate amounts to $680 \mu \mathrm{Gy} / \mathrm{h}$ corresponding to $6 \mathrm{~Gy} /$ year. Hence the total dose $D_{\text {total }}$

\footnotetext{
${ }^{6}$ Unfortunately no data are available, which allow to compare the attenuation lengths of identical scintillators in the years 1990 and 2007.
}

induced by the uranium plates within 17 years is

$D_{\text {total }}=(100 \pm 20) G y$.

The influence of radiation damage on the performance of an electromagnetic lead/scintillator calorimeter was studied with test beam measurements described in Refs. [6,8], which allows us to estimate the radiation dose which would be required to produce the same induced absorption as shown in Fig. 6 . The geometry of this test calorimeter was nearly identical to that of the electromagnetic section of the ZEUS FCAL. The main difference was that the $3.3 \mathrm{~mm}$ thick uranium plates were replaced by $6 \mathrm{~mm}$ thick lead plates. The scintillators and wave length shifters were identical to those of the electromagnetic sections of the ZEUS calorimeter. Prior to the beam test measurements the scintillators of different sections of the test calorimeter were irradiated uniformly with $\gamma$ rays at dose rates of about (100-200) Gy/h to doses of $D=0,5$ and $10 \mathrm{kGy}$, respectively. After the end of the recovery process in air only the permanent radiation damage remained. The properties of the test calorimeter were investigated in the DESY test beam with electrons of $1-6 \mathrm{GeV}$. Afterwards ${ }^{60} \mathrm{Co}$ measurements were performed, from which the optical parameters have been determined. The transmission $T$ is shown in Table 2 separately for electromagnetic sections EMC1/4 and for sections $\mathrm{EMC} 2 / 3$, because the geometry of the corresponding scintillators is slightly different. ${ }^{7}$ The absolute values $T$ obtained for non-irradiated scintillators of the test calorimeter are larger than the ones of the ZEUS calorimeter measured in 1990. A possible reason might be the different thickness of the absorber plates of the two calorimeters. Hence we compare only

\footnotetext{
7 The scintillators of sections EMC1/4 have two cutouts for spacers $\left(2.5 \times 9.8 \mathrm{~mm}^{2}\right)$.
} 
the relative values. The dose dependence of the ratio $T_{R}=$ $T(D) / T(D=0)$ measured in the test calorimeter is plotted in Fig. 7a. The solid curve presents a fit to the experimental data $\left(T_{R}=\exp (-\right.$ const $\left.\cdot D)\right)$. In the hadronic sections of the ZEUS calorimeter the transmission parameter decreased within 17 years by $(7.4 \pm 2.2) \%$ corresponding to the ratio $T(2007) / T(1990)=$ 0.926. As mentioned before, we have less results for the electromagnetic sections of the ZEUS calorimeter. But they show a very similar degradation. Due to the slightly different lengths of the scintillators in the hadronic and electromagnetic sections the absolute transmission parameters differ by $3 \%$. However, the relative change of the transmission is very similar for both parts of the calorimeter. This allows us to determine an equivalent dose $D_{\text {eq }}$ which would cause the same reduction of the transmission parameter as observed in the hadronic sections of the ZEUS calorimeter:

$D_{\text {eq }}=(2.8 \pm 1) \mathrm{kGy}$.
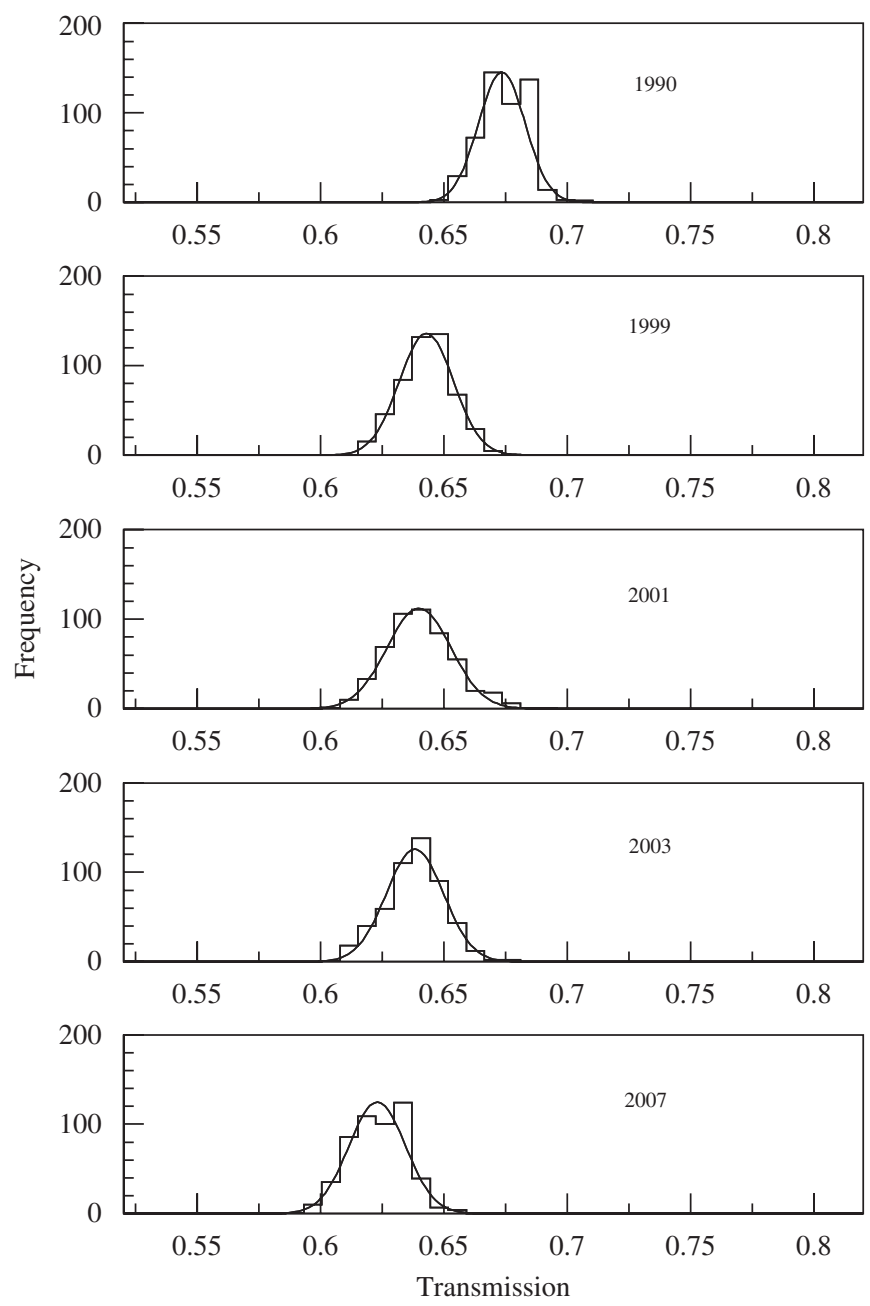

Fig. 5. Frequency distribution of transmission parameters $T$ of all scintillators of sections HAC1/2 of FCAL module 17 scanned in 1990, 1999, 2001, 2003 and 2007.
In order to determine the attenuation length of the scintillators SCSN-38 as a function of dose horizontal scans of the EMC sections were performed with $6 \mathrm{GeV}$ electrons. The test calorimeter was moved in steps of $3 \mathrm{~cm}$ parallel to the long side of the scintillators. For each position the light yields were measured with the left and right photomultipliers. After proper normalization the ratios of both signals were calculated for the sections EMC1-EMC4. From these results the attenuation lengths $\lambda$ presented in Table 2 and the corresponding absorption coefficients $\mu=1 / \lambda$ were derived. In the analysis the reflection of light at the scintillator edge opposite to the readout side was taken into account. Fig. 7b shows the variation of the absorption coefficient $\mu$ as a function of the dose $D$. For doses $D \leqslant 10 \mathrm{kGy}$ we assume that $\mu$ is a linear function of the dose. From both parts of Fig. 7 one can derive a relation between transmission parameter $T$ and radiation induced absorption coefficient $\mu$. The conclusion is that the exposure of the SCSN-38 scintillators to a dose of $(2.8 \pm 1) \mathrm{kGy}$ would reduce the transmission parameter of the test calorimeter by $(7.4 \pm 2.2) \%$ and increase the absorption coefficient by $(29 \pm 10) \%$.

\section{Summary and discussion}

${ }^{60}$ Co scans have been performed in the ZEUS calorimeter over a period of 17 years. No variation of the attenuation length of the polymethylmethacrylate based Y7 WLSs has been observed within this time. This is a very important result for ZEUS because the WLS bars have a length of up to $2 \mathrm{~m}$. After the assembling of the calorimeter modules the longitudinal uniformity of the calorimeter sections was in a range of $\pm 4 \%$. Any further decrease of the effective attenuation length would result in an increase of the non-linearity of the energy response of the calorimeter and would reduce the absolute light yield.

The absorption of fluorescence light in the polystyrene based scintillators (SCSN-38) increased continuously with time. The transmission $T$ decreased by $7.4 \%$ in 17 years. At medium dose rates $(\dot{D}=100-200 \mathrm{~Gy} / \mathrm{h})$ such an effect would occur after exposure to an equivalent dose $D_{\mathrm{eq}}=(2.8 \pm 1) \mathrm{kGy}$. This value is

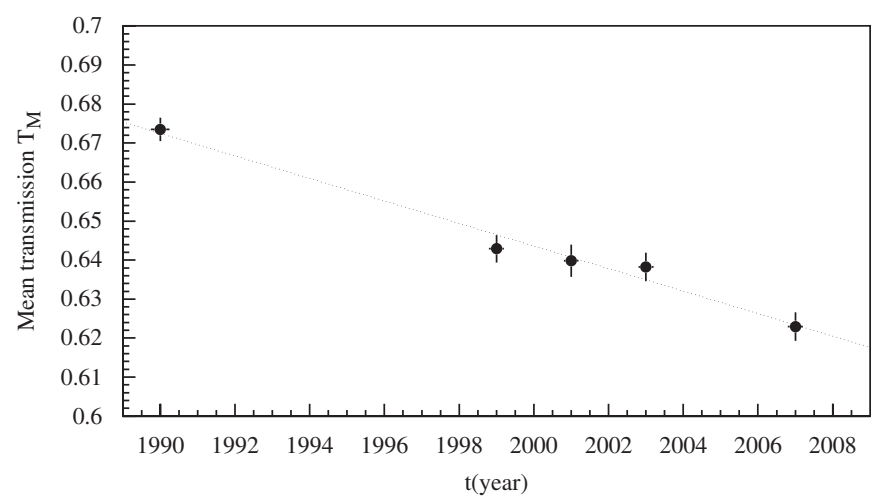

Fig. 6. Mean values of the Gaussian distributions of transmissions shown in Fig. 5. The errors are estimated to be $\Delta T=0.003$.

Table 1

Mean values of the transmission parameters $T$ for scintillators of the hadronic sections HAC1/2 of FCAL module 17 measured in $1990,1999,2001,2003$ and 2007.

\begin{tabular}{|c|c|c|c|c|c|}
\hline & 1990 & 1999 & 2001 & 2003 & 2007 \\
\hline Transmission of HAC scintillators & $0.673 \pm 0.009$ & $0.643 \pm 0.011$ & $0.640 \pm 0.013$ & $0.638 \pm 0.012$ & $0.623 \pm 0.012$ \\
\hline
\end{tabular}

The error calculation considers the sigma of the distribution as well as the number of contributing scintillators. 
Table 2

Mean value of the transmission parameters $T$ and attenuation lengths $\lambda$ for scintillators of the electromagnetic sections EMC1/2/3/4 of the test calorimeter.

\begin{tabular}{|c|c|c|c|c|}
\hline & Radiation dose (kGy) & 0 & 5 & 10 \\
\hline \multirow[t]{2}{*}{ EMC $1 / 4$ scintillators } & Transmission & $0.78 \pm 0.02$ & $0.65 \pm 0.02$ & - \\
\hline & Attenuation length (mm) & $664 \pm 57$ & $396 \pm 18$ & - \\
\hline \multirow[t]{2}{*}{ EMC $2 / 3$ scintillators } & Transmission & $0.72 \pm 0.01$ & - & $0.56 \pm 0.02$ \\
\hline & Attenuation length (mm) & $599 \pm 46$ & - & $318 \pm 11$ \\
\hline
\end{tabular}

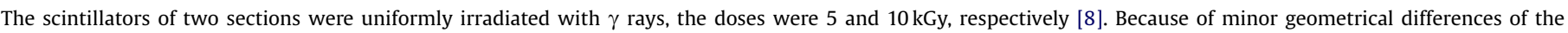
scintillators the sections EMC1/4 and EMC 2/3 are considered separately.

a

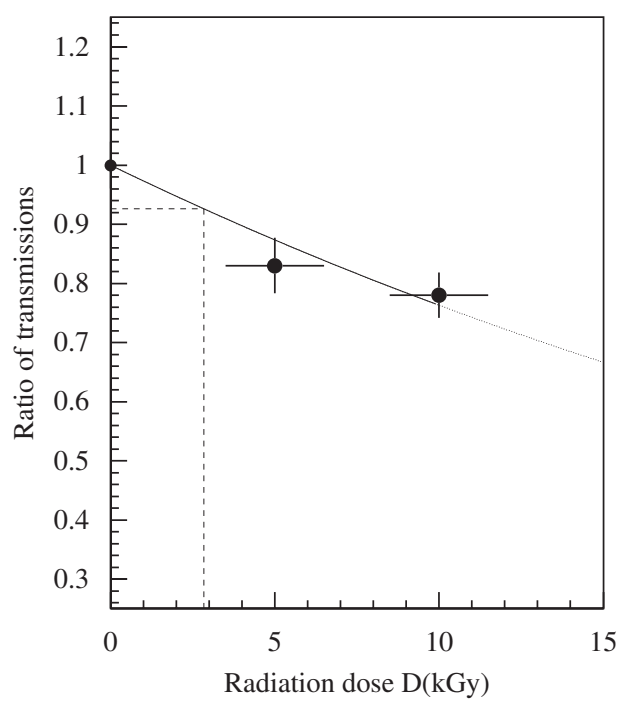

b

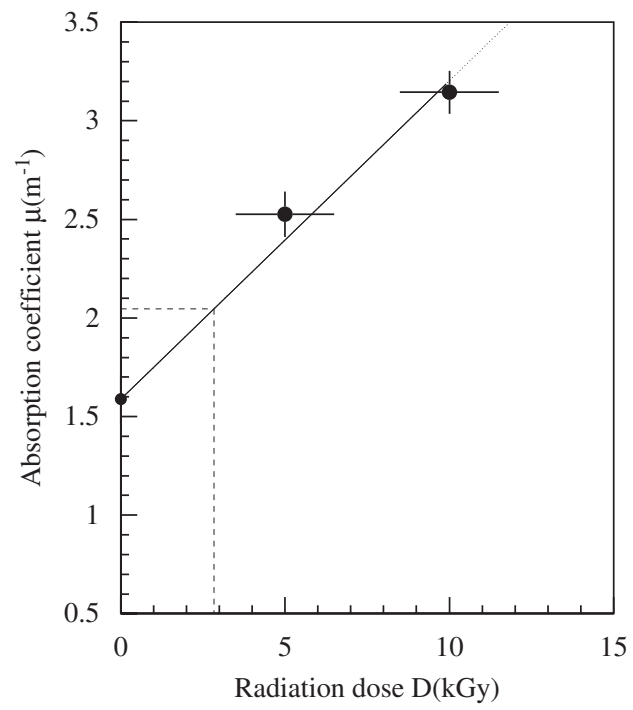

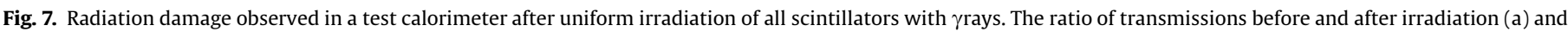

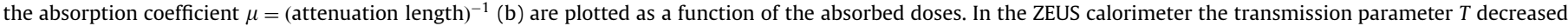

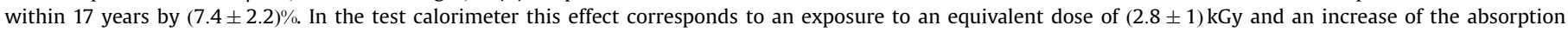
coefficient by $(29 \pm 10) \%$.

much larger than the actual dose $D_{\text {total }}=(100 \pm 20)$ Gy deposited in the scintillators within a period of 17 years. This dose was caused by the radioactivity of the uranium plates at a very low dose rate $(680 \pm 140 \mu \mathrm{Gy} / \mathrm{h})$.

In the following it will be discussed whether the damage observed in the ZEUS calorimeter is caused by radiation or ageing. Let us first assume that is caused by radiation. In the ZEUS and the test calorimeter we found the same damage effects at quite different doses and dose rates. This is only possible when radiation damage in SCSN-38 is strongly dose rate dependent. At high doses $(D=25 \mathrm{kGy})$ and dose rates in the range $\dot{D}=$ $30-1000 \mathrm{~Gy} / \mathrm{h}$ no dose rate effect has been observed in SCSN-38 $[9,10]$. Only a few investigations concerning radiation damage at low doses and low dose rates have been performed [11,12]. Since the damage effects under these conditions are very small, the authors used scintillating fibres to study the time dependence of the radiation induced absorption during and after irradiation. They investigated fibres (SCSF-38 and SCSF-38M) of about $1 \mathrm{~m}$ length, both fibres had a polystyrene core and the same fluors as SCSN-38. The M in SCSF-38M indicates that the fibre had double cladding. In Ref. [11] the fibres SCSF-38 were exposed to X-rays, the dose rate and the maximum dose were $36 \mathrm{~Gy} / \mathrm{h}$ and $2175 \mathrm{~Gy}$, respectively. In Ref. [12] the fibres SCSF-38M were irradiated with $\gamma$ rays from a ${ }^{137} \mathrm{Cs}$ source, the dose rate and the maximum dose were $(0.97 \pm 0.11) \mathrm{Gy} / \mathrm{h}$ and $160 \mathrm{~Gy}$, respectively. No evidence for a dose rate effect has been found within the experimental errors.

The conclusion of the discussion is that the damage observed in the ZEUS calorimeter is not caused by radiation, neither by the beams nor by the radioactivity of DU. It is most likely due to ageing of the scintillators. Ageing effects in SCSN-38 have been investigated in Ref. [13]. The authors have studied the stability of several plastic scintillators of $1 \mathrm{~m}$ length over a period of 4 years. For SCSN-38 they found that the attenuation length $\lambda$ decreased by $(8 \pm 3.1) \%$ within 1000 days (2.7 years). The absorption coefficient $\mu$ is proportional to the concentration of absorption centres. Hence we assume that $\mu$ (and not the attenuation length ג) rises linearly with time:

$\mu(t)=\alpha+\beta t$.

From the results of Ref. [13] one obtains $\alpha=(1.22 \pm 0.02) \mathrm{m}^{-1}$ and $\beta=(3.8 \pm 1.6) \times 10^{-2} \mathrm{~m}^{-1} \mathrm{yr}^{-1}$. If one extrapolates Eq. (4) to $t=$ 17 years, one obtains a large increase of the absorption coefficient $(\Delta \mu / \mu \approx(53 \pm 22) \%)$. At the ZEUS calorimeter we have measured the transmission parameter $T$ and not the absorption coefficient $\mu$ or the attenuation length $\lambda$. The relation between $T$ and $\mu$ was determined for radiation damage. In the following we assume that the same relation holds for ageing effects as well (see Fig. 7). This means that the damage observed in the ZEUS calorimeter corresponds to an increase of the absorption coefficient by $(29 \pm 10) \%$. The result is smaller than that obtained from Eq. (4), but within the experimental uncertainties both results are compatible. This can be considered as a further confirmation that the damage observed in the ZEUS calorimeter can be interpreted as an ageing effect.

The degradation of the attenuation lengths of the scintillators in the ZEUS calorimeter is not a problem for the detector 
performance. For the test calorimeter it has been shown in Ref. [8] that effects of the size observed in the ZEUS calorimeter have no influence on the energy resolution and the linearity of the energy response of the electromagnetic calorimeter. For two reasons the calorimeter performance was stable although the attenuation length of the scintillators changed: the length of the scintillators $(L \approx 19 \mathrm{~cm})$ was much shorter than the attenuation length $(\lambda \approx 50-70 \mathrm{~cm})$, so that the variation of the attenuation length had no significant consequences. The mean path length of the fluorescence light in the scintillators was further reduced because the light was read out at both edges of the scintillators.

\section{Acknowledgements}

A large number of physicists and technicians worked for this long-term study within the last 17 years. We thankfully acknowledge the contributions of B. Böhnert, G. Gloth, W. Grell, A. Hacker, A. Henkel, M. Herrmann, S. Jacobs, B. Krebs, R. Krüger, D. Kummerow, F. Meyer, Th. Neumann, T. Rimkus. We also want to thank the ZEUS calorimeter group for their continuous support and useful discussions, especially W. Hain, R. Klanner, U. Schneekloth, T. Tsurugai, R. Yoshida, and W. Zeuner. It is a pleasure to thank K. Flöttmann for a critical reading of the whole draft. We would like to thank the referee for his proposals which improved the manuscript considerably.

\section{References}

[1] I. Bloch, et al., IEEE Trans. Nucl. Sci. NS-51 (2004) 1606.

[2] U. Behrens, et al., Nucl. Instr. and Meth. A323 (1992) 611.

[3] I. Bohnet, N. Gendner, F. Goebel, T. Neumann, K. Wick, Radiation hardness of different calorimeters studied with a movable ${ }^{60} \mathrm{Co}$ source at the ZEUS detector, in: Proceedings of the 8th International Conference on Calorimetry in High Energy Physics, Lisbon, Portugal, World Scientific, Singapore, 1999, p. 639.

[4] T. Kamon, K. Kondo, A. Yamashita, T. Shimizu, L. Nodulman, Nucl. Instr. and Meth. 213 (1983) 261.

[5] A. Andresen, et al., Nucl. Instr. and Meth. A309 (1991) 101.

[6] I. Bohnet, Investigations on uniformity, radiation resistance and calibration of ZEUS calorimeter components at HERA, Ph.D. Thesis, University of Hamburg, DESY-Thesis-1999-041, December 1999.

[7] B. Anders, H. Brückmann, Radiation damage of organic scintillators from natural depleted uranium (DU) radioactivity, Annual Report 1984/1985, I. Institut für Experimentalphysik, University of Hamburg, p. 75.

[8] I. Bohnet, D. Kummerow, K. Wick, Nucl. Instr. and Meth. A490 (2002) 90.

[9] K. Wick, D. Paul, P. Schröder, V. Stieber, D. Bicken, Nucl. Instr. and Meth. B61 (1991) 472.

[10] U. Holm, K. Wick, IEEE Trans. Nucl. Sci. NS-36 (1989) 579.

[11] Th. Zoufal, Beeinträchtigung der optischen Eigenschaften und Veränderung der Fluoreszenz von Kunststoffszintillatoren durch Röntgenstrahlung, Ph.D. Thesis, University of Hamburg, 2002.

[12] A. Ziegler, Investigation of the scintillating fiber SCSF-38M for the $6 \mathrm{~m}$-tagger and measurement of inclusive $K_{S}^{0}$ meson and $\Lambda$ baryon production in neutral current deep inelastic scattering at HERA, Ph.D. Thesis, University of Hamburg, DESY-Thesis-2003-012, 2003.

[13] T. Hasegawa, et al., Nucl. Instr. and Meth. A311 (1992) 498 